\title{
Plain-Language Summaries: An Essential Component to Promote Knowledge Translation
}

\author{
Sai Krishna Gudi ${ }^{1}$, Komal Krishna Tiwari ${ }^{1}$, and Kainat Panjwani ${ }^{2}$ \\ ${ }^{1}$ University of Manitoba \\ ${ }^{2}$ Marri Laxman Reddy Institute of Technology and Management
}

November 7, 2020

\begin{abstract}
In this era of evidence-based practice, scholarly work such as peer-reviewed scientific publications play a vital role in policy and decision-making at an individual, organization, and country-level. Alongside being considered an essential means of communicating scholarly work, scientific publications also investigate the specific domains that lack well-established literature and thereby inform scientists and researchers to thrive for the betterment of the publics' well-being. Thus, the main purpose of articulating the scientific, scholarly work should be to make it understandable and accessible to everyone, including the lay audience. However, oftentimes, researchers overlook the lay summaries while publishing the research findings.
\end{abstract}

\section{Hosted file}

IJCP.pdf available at https://authorea.com/users/373910/articles/491523-plain-languagesummaries-an-essential-component-to-promote-knowledge-translation 